Journal for

... ImmunoTherapy of Cancer

\section{Malignant cell-specific CXCL14 promotes tumor lymphocyte infiltration in oral cavity squamous cell carcinoma}

Anuraag Parikh (D) , ${ }^{1}$ JuneHo Shin, ${ }^{2}$ William Faquin, ${ }^{3}$ Derrick T Lin, ${ }^{1}$ Itay Tirosh, ${ }^{4}$ John B Sunwoo, ${ }^{2}$ Sidharth V Puram ${ }^{5,6}$
To cite: Parikh A, Shin J, Faquin W, et al. Malignant cell-specific CXCL14 promotes tumor lymphocyte infiltration in oral cavity squamous cell carcinoma. Journal for ImmunoTherapy of Cancer 2020;8:e001048. doi:10.1136/ jitc-2020-001048

- Additional material is published online only. To view, please visit the journal online (http://dx.doi.org/10.1136/jitc2020-001048).

AP and JS contributed equally.

Presented as podium presentation at: American Academy of OtolaryngologyHead and Neck Surgery Annua Meeting, September 15-18, 2019, New Orleans, Louisiana, USA.

Accepted 23 July 2020

Check for updates

(C) Author(s) (or their employer(s)) 2020. Re-use permitted under CC BY-NC. No commercial re-use. See rights and permissions. Published by BMJ.

For numbered affiliations see end of article.

\section{Correspondence to}

Professor Sidharth V Puram; sidpuram@wustl.edu

Professor John B Sunwoo; sunw00@stanford.edu

\section{ABSTRACT}

Objectives To explore lymphocyte infiltration as a potential mechanism behind CXCL14-mediated tumor growth suppression in oral cavity squamous cell carcinoma (OSCC).

Methods We analyzed single cell RNA-sequencing (scRNA-seq) data from OSCC to identify expression changes among malignant cells in lymph nodes (LN) versus primary tumors. CXCL14 expression in murine OSCC cell lines was quantified using qRT-PCR. Short hairpin RNA knockdown of CXCL14 was performed in mouse oral cavity (MOC)1 cells, and CXCL14 overexpression was performed in MOC2 cells. Cells in each condition were injected into C57BL/6 mice with and without T cell depletion, and tumor volume was measured. At 30 days, tumors were dissociated and analyzed by flow cytometry for $\mathrm{CD} 45^{+} \mathrm{CD} 3^{+}$ T cells. CXCL14 expression was correlated with gene expression signatures of tumor infiltrating lymphocytes (TIL) in scRNA-seq data, as well as TCGA tumors.

Results scRNA-seq revealed CXCL14 as the most significantly downregulated gene among malignant cells in LNs relative to primary tumor, supporting a role in preventing invasion and/or metastasis. In a murine immunocompetent model, CXCL14 expression was higher in indolent MOC1 cells than in more aggressive MOC2 cells. Tumor growth in vivo was significantly increased by CXCL14 knockdown in MOC1 cells relative to control, with a corresponding decrease in TIL. In MOC2 cells, tumor growth was significantly reduced by CXCL14 overexpression relative to control and TIL were increased. Both effects were lost with T cell depletion. In a human tumor scRNA-seq cohort, we found that only malignant cell CXCL14, but not non-malignant cell or fibroblast CXCL14, was associated with TIL. Bulk CXCL14 from the TCGA cohort had no association with TIL. Conclusions Higher CXCL14 expression by tumor cells is associated with reduced tumor growth and increased TIL, supporting immune-mediated suppression of tumor growth in OSCC. Given that CXCL14 is downregulated in LN metastases compared with primary tumors, our data raise the possibility that CXCL14-mediated immune infiltration may discourage invasion and metastasis. In human scRNA-seq data, only malignant cell-specific CXCL14 was associated with TIL, suggesting a critical context-dependent effect of CXCL14 expression.

\section{INTRODUCTION}

CXCL14 is a cytokine with pleiotropic functions ${ }^{1}$ and varying effects on tumor growth across tumor types. ${ }^{2-6}$ In head and neck squamous cell carcinoma (HNSCC), CXCL14 expression has been demonstrated to be reduced in tumor tissues relative to adjacent normal or oral dysplasia tissues. ${ }^{7}$ CXCL14 has also previously been associated with decreased growth in HNSCC xenograft models, ${ }^{8-10}$ supporting its role as a tumor suppressor in HNSCC. This decreased expression has been attributed to epigenetic silencing of CXCL14 by promoter hypermethylation in tumor tissues, ${ }^{71}$ and CXCL14 expression was shown to be restored by treatment with 5-azacytidine, ${ }^{712}$ a demethylating agent.

Multiple studies have assessed the impact of CXCL14 on growth. Ozawa et $a l^{8}$ demonstrated that in vitro growth of CXCL14overexpressing cells is not suppressed, suggesting that tumor suppression is not related to intrinsic cellular growth retardation. Tessema et $a l^{12}$ demonstrated increased cell death in vitro and reduced tumor growth via increased necrosis in vivo of lung tumor xenografts after forced expression of CXCL14 supporting the hypothesis that CXCL14mediated tumor suppression may be related to anti-angiogenic activity. ${ }^{13}$

Other studies have investigated the role of this chemokine on immune cell infiltration. Shurin et $a l^{14}$ demonstrated that CXCL14 may be chemotactic for immature dendritic cells (DC) and proposed that this may be one mechanism for its effects. Cicchini et al ${ }^{15}$ demonstrated that CXCL14 expression is downregulated during human papillomavirus (HPV)-associated head and neck and cervical cancer progression, and re-expression of CXCL14 induces infiltration of natural killer cells and $\mathrm{T}$ cells into tumor draining lymph nodes. The group subsequently demonstrated that CXCL14 expression induces $\mathrm{T}$ cell activation and restores major histocompatibility complex (MHC) class I expression in HPV-associated head and neck cancer 
models, leading to tumor cell death. ${ }^{16}$ Unfortunately, a detailed understanding of CXCL14 and its secretory origin in HPV-negative oral cavity squamous cell carcinoma (OSCC), the most common form of HNSCC, has remained unknown.

Here, we examined the relationship between CXCL14 and tumor infiltrating lymphocytes (TIL) in human HNSCC, and using murine immunocompetent models of HPV-negative OSCC, validated the ability of CXCL14 to induce TIL accumulation in the tumor microenvironment and inhibit tumor growth in vivo.

\section{METHODS}

\section{Analysis of single cell RNA-seq data}

Single cell RNA-sequencing (scRNA-seq) data were obtained from and re-analyzed as described in Puram $e t$ $a l .{ }^{17}$ For five samples with matched primary OSCC tumors and metastatic lymph node samples (MEEI5, MEEI20, MEEI25, MEEI26 and MEEI28), differential expression between primary tumor and lymph node niches was analyzed. Equal numbers of cells were sampled from each patient to ensure no bias towards a particular patient's data, and sampling was done 100 times to calculate a p value. For nine primary tumor samples (MEEI5, MEEI16, MEEI17, MEEI18, MEEI20, MEEI22, MEEI25, MEEI26 and MEEI28), average CXCL14 and MHC class I expression in malignant cells were determined.

\section{Mice and cell lines}

C57BL/6 mice were obtained from Jackson Laboratory (Bar Harbor, Maine, USA). Mice were kept under specific pathogen-free conditions, and mice aged $6-8$ weeks were used for all experiments. Mouse oral cavity (MOC) 1 and MOC2 murine oral squamous cell carcinoma cell lines, developed from murine oral squamous cell carcinomas induced by topical 7,12-dimethylbenz(a)anthracene administration, were generously provided by Dr Ravindra Uppaluri at Washington University (St. Louis, Missouri, USA). Cells were cultured in complete DMEM/ F-12 medium (Mediatech) containing 10\% fetal bovine serum (FBS) and 1\% penicillin and streptomycin. Cells were maintained at $37^{\circ} \mathrm{C}$ in a humidified atmosphere containing $5 \% \mathrm{CO}_{2}$.

\section{Organoid culture}

For mouse-derived organoids, tongue tissue was obtained from C57BL/6 mice. Excess fat and muscle tissue were removed to enrich for epithelial cells. The tissue was then finely minced into $2-3 \mathrm{~mm}$ pieces on ice, washed twice in DMEM/F-12 (Invitrogen) containing 1X Primocin (InvivoGen), followed by ACK solution (Thermo Fisher Scientific) to remove red blood cell contamination. Minced tissues were washed with DMEM/F-12 media and resuspended with Matrigel (cold Cultrex growth factor reduced Basement Membrane Extract (BME) type 2, Trevigen). Droplets of approximately $40 \mu \mathrm{L}$ were plated on the bottom of preheated 24-well culture plates $(\mathrm{E} \& \mathrm{~K}$
Scientific). Plates were incubated at $37^{\circ} \mathrm{C}$ for $30 \mathrm{~min}$ to allow BME to solidify. Organoid culture media (EN media) containing DMEM/F-12 supplemented with $10 \%$ Noggin-conditioned media, nicotinamide $(10 \mathrm{mM}$, Sigma), N-acetylcysteine (1 mM, Sigma), B-27 without vitamin A (1X, Invitrogen), Pen-Strep (1X, Invitrogen) and EGF (50 ng/mL, R\&D Systems) was added. The medium was changed every 2-3 days and organoids were split once every $2-4$ weeks.

\section{qRT-PCR of CXCL14 in cell lines}

RNA was extracted with the RNeasy mini kit (QIAGEN) and complementary DNA (cDNA) libraries were prepared with the Maxima First Strand cDNA Kit (Thermo Fisher Scientific). Gene expression probes for mouse CXCL14 (Mm00444699_m1, Thermo Fisher Scientific) and mouse HPRT1 (Mm03024075_m1, Thermo Fisher Scientific) were purchased. Quantitative gene expression was performed using the Taqman Gene Expression Assay with recommended primers (Life Technologies). Gene expression was normalized to control for HPRT1 expression and then shown relative to an appropriate control $(2 \Delta \mathrm{Ct} \times 100$, where $\Delta \mathrm{Ct}$ represents the difference in threshold cycle between the control and target genes).

\section{Lentiviral plasmids}

RNAi Consortium (TRC) Lentiviral short hairpin RNA (shRNA) (pLKO.1-based vector) was purchased from GE Healthcare Dharmacon. A cDNA encoding CXCL14 from ATG codon to the stop codon was PCR cloned (Forward primer: 5'-GAATTC ATGAGGCTCGTGGCGGCCG-3'; reverse primer: 5'-GGATCC CTATTCTTCGTAGACCCT- $3^{\prime}$ ) from pCMV6-cxcl14 (Origene) and subcloned EcoRI/BamHI fragments into the pHIVZsgreen lentiviral expression construct (Addgene).

\section{Lentiviral production and transduction}

For the production of the lentiviral particles, the 293 $\mathrm{T}$ cell line was transfected with the packaging plasmid pCMVR8.74, the envelope plasmid pCMV-VSVG and the lentiviral construct containing the shRNA or the transgene, using Lipofectamine 2000 according to the manufacturer's instructions. The medium was changed 16 hours after the transfection. Virus-containing culture supernatant was collected after 24 hours and centrifuged for concentration. Virus was used immediately to infect cells, which were seeded at $3 \times 10^{5}$ cells per well in a 6 -well plate 24 hours prior. Polybrene $(8 \mu \mathrm{g} / \mathrm{mL})$ was also added to enhance the lentiviral transduction efficiency. The medium was changed after 24 hours. In the case of the MOC1 cells transduced with the pLKO.1 puro vectors, the cell cultures were treated with $1 \mu \mathrm{g} / \mathrm{mL}$ puromycin for 1 week after media change. For pHIV-Zsgreen transduced MOC2 cells were sorted (GFP-positive) with FACSAria machine (BD Biosciences).

\section{Cell proliferation assay}

Proliferation experiments were conducted using $x C E L$ Ligence RTCA DP device (ACEA Biosciences, San Diego, 
California, USA), which was placed in a humidified incubator at $37^{\circ} \mathrm{C}$ in $5 \% \mathrm{CO}_{2}$. Cell proliferation experiments were carried out using 96-well plates. Microelectrodes for impedance detection during cell attachment, spreading, and proliferation were attached at the bottom of each well and had electronic connection with the computer software. At the beginning, $100 \mu \mathrm{L}$ complete growth medium was added to each well, and water was added to the space around the wells to avoid evaporation. Plates were incubated for 30 minutes at room temperature in a laminar chamber. Afterwards, incubation plates were inserted into the device and the background impedance was measured. Next, the MOC1 and MOC2 cells were seeded $1 \times 10^{4}$ cells/well in $100 \mu \mathrm{L}$ growth medium per well. Plates were left for 30 minutes at room temperature in a laminar chamber to allow for cell attachment. Finally, the plates were inserted into the device and impedance was automatically monitored and expressed as the Cell Index (CI) value by the software. Cell proliferation experiments were run for 60 hours. CI was monitored every $15 \mathrm{~min}$ for the whole experiment duration.

\section{Transwell invasiveness assay}

To assess the invasive capacity of the tumor cells, $1 \times 10^{4}$ cells in $500 \mu \mathrm{L}$ of serum-free DMEM/F-12 were added into the upper chamber and $500 \mu \mathrm{L}$ of complete medium was placed into the lower chamber (Corning BioCoatTM Matrigel Invasion Chamber, Corning, New York, USA). Cells were incubated at $37^{\circ} \mathrm{C}$ for 48 hours, before the non-invading cells were removed from the upper surface of the membrane. After fixation in 95\% ethanol for 5 minutes, the cells still on the opposite surface of the filter membrane were stained with $1 \%$ crystal violet for 10 minutes. The migratory cells were counted in five microscope fields and averaged.

\section{Analysis of MHC class I expression}

To assess the expression of MHC class I genes, samples were stained with $\mathrm{PE}-$-conjugated antimouse $\mathrm{H}-2 \mathrm{~K}^{\mathrm{b}}$ antibody (clone AF6-88.5, Biolegend) and Alexa Fluor 647 antimouse $\mathrm{H}_{-2} \mathrm{D}^{\mathrm{b}}$ antibody (clone KH95, Biolegend) and analyzed by flow cytometry.

\section{In vivo mouse tumor growth assays}

$10^{6}$ cells in each condition were injected into immunocompetent C57BL/6 mice $(\mathrm{n}=16$; control and experimental group cells were injected into the left and right flank, respectively), and tumor volume was measured at the time points shown.

\section{Tumor digestion and flow cytometry analysis}

For fluorescent activated cell sorting (FACS) analysis, tumors were harvested, minced and digested in $300 \mathrm{U} /$ $\mathrm{mL}$ collagenase and $100 \mathrm{U} / \mathrm{mL}$ hyaluronidase (StemCell Technologies) in culture media: DMEM/F-12 medium (Mediatech) with 10\% FBS, $2 \mathrm{mmol} / \mathrm{L}$ L-glutamine and $1 \%$ penicillin-streptomycin-amphotericin B (MP Biomedicals). The tumor digest was pipetted every $15 \mathrm{~min}$ and incubated at $37^{\circ} \mathrm{C}$ for 3 hours, until a single-cell suspension was obtained. The dissociated cells were resuspended in Trypsin-EDTA (StemCell Technologies) for $5 \mathrm{~min}$, then further dissociated with $5 \mathrm{U} / \mathrm{mL}$ dispase (StemCell Technologies) and $0.1 \mathrm{mg} / \mathrm{mL}$ DNase I (StemCell Technologies) for $1 \mathrm{~min}$. Cells were filtered through a $40 \mathrm{~mm}$ cell strainer and erythrocytes were lysed with ACK lysing buffer (Lonza). Suspensions were then pretreated with IgG from mouse serum to block non-specific staining and incubated with anti-CD45 and CD3 antibodies (Biolegend). 4',6-diamidino-2-phenylindole (DAPI) was used to allow exclusion of non-viable cells. FACS analysis was performed with FACSAria (BD Biosciences).

\section{T cell depletion}

T cells were depleted using a CD3 antibody. The following antibodies were used: hamster IgG $\mathrm{f}(\mathrm{ab}$ ') two fragment control antibody (clone BE0091-FAB f(ab'), Bio X Cell) and CD3-depleting antibody (clone 125-2C11 f(ab') two fragments). Mice were treated on days -2 and 1 via intraperitoneal inoculation with $200 \mu \mathrm{g}$ of CD3-depleting antibody diluted in phosphate-buffered saline to a final volume of $200 \mu \mathrm{L}$.

\section{Histological assessment of lymphocytes in scRNA-seq cohort}

H\&E slides of nine primary tumors analyzed by scRNA-seq ${ }^{17}$ were obtained and analyzed in a blinded manner by a dedicated head and neck pathologist (WF). Whole slides were analyzed for stromal lymphocytes adjacent to tumor nests. Stromal lymphocytic infiltrate was qualitatively scored as 1+ (absent or minimal), 2+ (moderate) or 3+ (dense). For each sample, TIL were also assessed as follows: whole slides were analyzed to identify the regions of highest TIL density. In these regions, TIL were calculated on five high power fields (HPFs) with a $40 \times$ objective. Slides were visualized on an Olympus BX40CY microscope at $400 \times$. Images were taken at $40 \times$ on an Olympus DP27 camera.

\section{Analysis of TCGA data for CXCL14 and TIL}

Bulk transcriptomic data for 498 OSCC tumors (rnaseqv2RSEM_genes_normalized) was downloaded from the Broad Firehose website (https://gdac.broadinstitute. org/), along with additional tumor and clinical annotations. Expression data were $\log _{2}$-transformed and centered for each gene. The relative abundance of TIL in each tumor was estimated as the average normalized expression of CD2, CD3D, CD3E and CD3G. These estimates were evaluated by the Pearson's correlation with the normalized expression of CXCL14.

\section{RESULTS}

\section{CXCL14 is downregulated in lymph nodes}

Malignant cells in primary tumor and lymph node niches were analyzed for five matched samples previously profiled by scRNA-seq. ${ }^{17}$ A number of genes were differentially expressed in one or more patients (figure 1). Focusing on genes that demonstrated concordant differential 


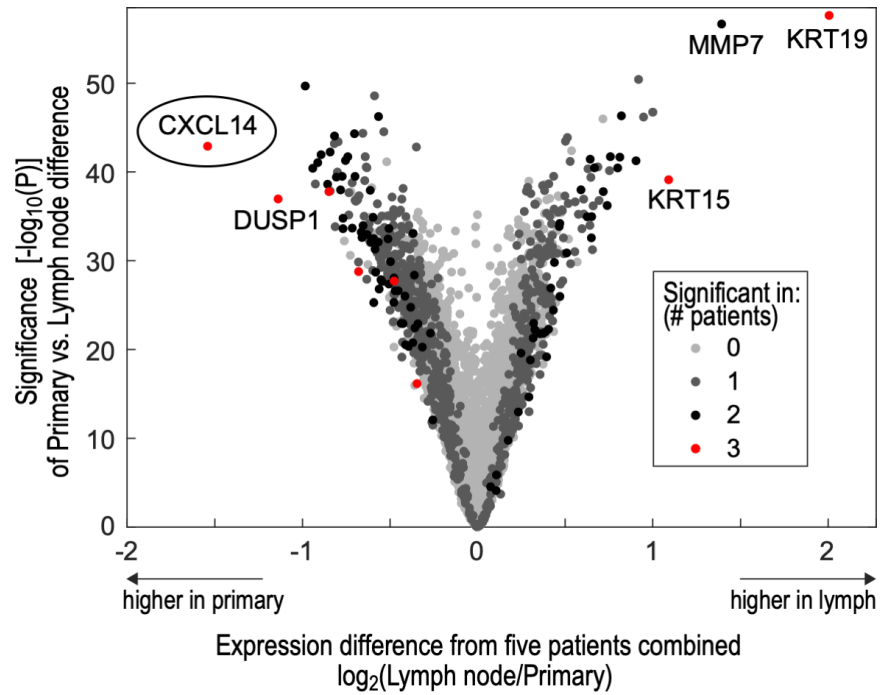

Figure 1 Differential gene expression between primary tumors and lymph nodes shows CXCL14 as the top downregulated gene in metastases. Volcano plot shows differentially expressed genes in five primary tumor samples relative to matched metastatic cervical lymph nodes, in malignant cells profiled by single cell RNA-sequencing. Genes are plotted based on differential expression (x-axis) and significance of this difference (y-axis). They are also colored based on the number of individual patient samples in which differential expression was significant. CXCL14 was the most significantly downregulated gene across lymph node samples and demonstrated significant downregulation in three individual patients.

expression in the majority of samples, we found that CXCL14, DUSP1, NFKBIA, FOS and HSPA1B were significantly downregulated and KRT19 and KRT15 were significantly upregulated in the lymph nodes of three out of five samples (figure 1). Of these, CXCL14 was the most highly downregulated gene (2.9-fold change, $\mathrm{p}=10^{-43}$ ) in lymph nodes relative to primary tumors, suggesting it may play a role in suppressing metastasis in HNSCC. Given the previously described associations of CXCL14 with reduced tumor growth in HNSCC, ${ }^{8-10}$ we focused further investigations on the role of this chemokine as a tumor suppressor and, in particular, on its ability to induce the host immune response.

\section{CXCL14 expression is lower in aggressive cell lines}

CXCL14 is expressed constitutively in normal squamous epithelial cells but heterogeneously in most HNSCC and cervical SCC tumors. ${ }^{18}$ To understand the function of CXCL14 in OSCC, CXCL14 expression was analyzed in MOC1 and MOC2 cell lines relative to housekeeping gene HPRT. MOC1 and MOC2 cell lines were used because of previously demonstrated differences in MOC tumor aggressiveness and immune infiltration. Analysis of transcripts showed that CXCL14 is expressed in both cell lines but at a significantly higher level in indolent MOC1 cells $(0.12 \pm 0.011)$, relative to aggressive MOC2 cells $(0.03 \pm 0.004)$, with approximately a fourfold change between the cell lines $(\mathrm{p}<0.05, \mathrm{n}=3$ biological replicates $)$
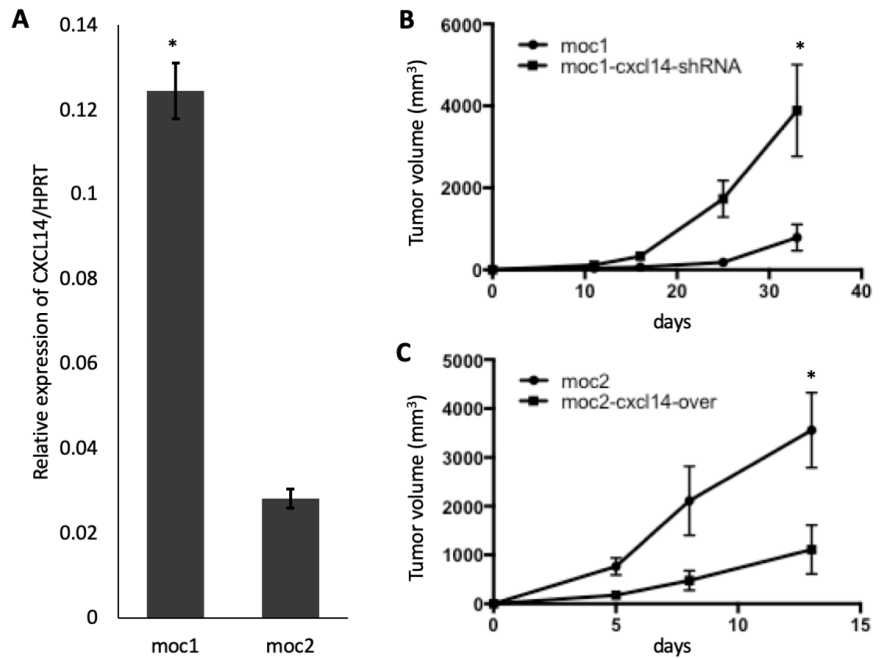

Figure 2 Modulation of CXCL14 is associated with in inverse changes in tumor size. (A) Bar plot shows expression of CXCL14 relative to housekeeping gene HPRT in multiple replicates $(n=3$ biological replicates, 2 independent experiments) in murine immunocompetent models. CXCL14 was significantly higher in indolent mouse oral cavity (MOC) 1 cells than in more aggressive MOC2 cells. (B) Line plot shows significant increase in MOC1 derived tumor growth after CXCL14 shRNA knockdown ( $n=5$ biological replicates, 2 independent experiments, ${ }^{*} \mathrm{p}<0.05$; error bars represent SEM). (C) Line plot shows significant decrease in MOC2derived tumor growth after CXCL14 overexpression $(n=5$ biological replicates, 2 independent experiments, ${ }^{*} p<0.05$; error bars represent SEM).

(figure 2A). These findings indicate that CXCL14 expression may be correlated with indolent, rather than aggressive, HNSCC tumors.

\section{In vivo tumor growth is inversely correlated with CXCL14}

To determine whether CXCL14 expression is associated with in vivo tumor growth, we performed subcutaneous tumor growth assays with MOC1 and MOC2 cell lines in immunocompetent C57BL/6 mice. To examine the specific function of CXCL14 in growth of MOC tumors, CXCL14 expression was inhibited in MOC1 cells by stably transducing an shRNA targeting CXCL14 (MOC1CXCL14-shRNA) and overexpressed in MOC2 cells by stably transducing a CXCL14 expression construct (MOC2-CXCL14-over) (online supplemental figure S1). The degree to which CXCL14 was expressed in these cell lines was found to correlate with tumor growth kinetics in vivo. Tumor growth from these cells when injected into immunocompetent syngeneic mice was significantly higher with MOC2 cells than with MOC1 cells, at baseline (figure 2B,C, $\mathrm{p}<0.05$ ). Relative to control, injection of MOC1 cells after CXCL14 shRNA knockdown resulted in significantly larger tumors (figure $2 \mathrm{~B}, 5.80$-fold, $\mathrm{p}<0.05$, $\mathrm{n}=5$ ), and conversely, injection of MOC2 cells after CXCL14 overexpression resulted in significantly smaller tumors (figure 2C, 5.88-fold, $\mathrm{p}<0.05, \mathrm{n}=5$ ). These results suggest a potential tumor suppressive effect of CXCL14 in vivo. 
A

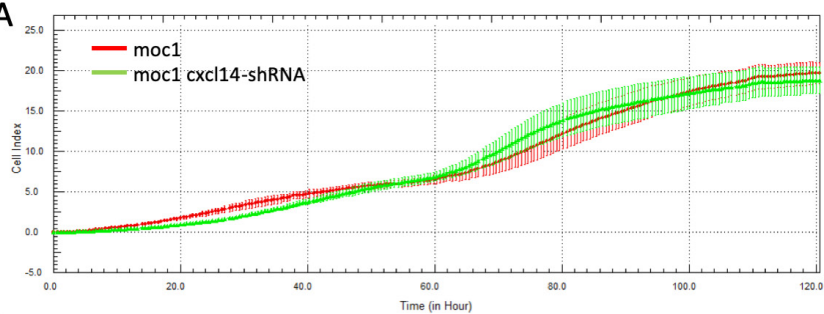

B

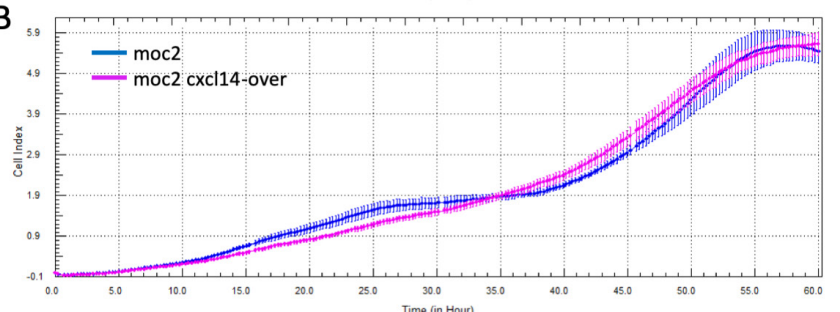

C

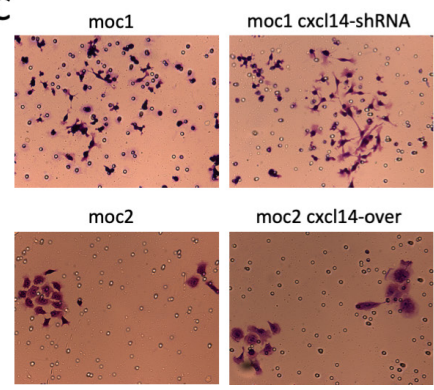

$D_{70}$

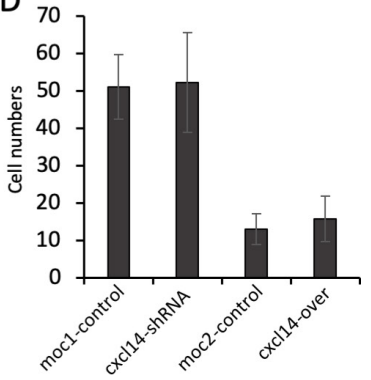

Figure 3 Modulation of $\mathrm{CXCL} 14$ is not associated with in vitro changes in proliferation or invasiveness. $(A)$ and $(B)$ Line plots show growth curves of mouse oral cavity (MOC) 1 and $\mathrm{MOC} 2$ cells in control and experimental conditions using the $x$ CELLigence system. There were no significant changes in growth with modulation of CXCL14. (C) Photos show representative images of crystal violet stained cells from transwell assay. (D) Bar plots show numbers of invaded cells (error bars represent SEM). MOC1 cells were more invasive than MOC2 cells, but CXCL14 modulation was not associated with changes in invasiveness of either cell line.

\section{In vitro proliferation and invasiveness are not correlated with CXCL14}

To determine whether changes in in vivo tumor growth in response to CXCL14 modulation were attributable to tumor cell-intrinsic changes, proliferation and invasiveness were assessed in vitro. CXCL14-modulated MOC cells showed no significant difference in in vitro cell proliferation capacity (figure 3A,B) or invasiveness (figure 3C,D), suggesting that in vivo tumor growth differences are related to CXCL14 effects on the tumor microenvironment.

\section{CXCL14 expression is associated with increased TIL}

We next sought to determine whether modulation of tumor CXCL14-dependent growth may be associated with immune cell infiltration. To this end, we quantified tumor infiltrating $\mathrm{T}$ cells after injection of MOC cells into immunocompetent syngeneic mice. Tumors were harvested and analyzed by flow cytometry for $\mathrm{CD} 45^{+} \mathrm{CD}^{+} \mathrm{T}$ cells. Tumors generated following CXCL14 shRNA knockdown in MOC1 cells demonstrated an $82 \%$
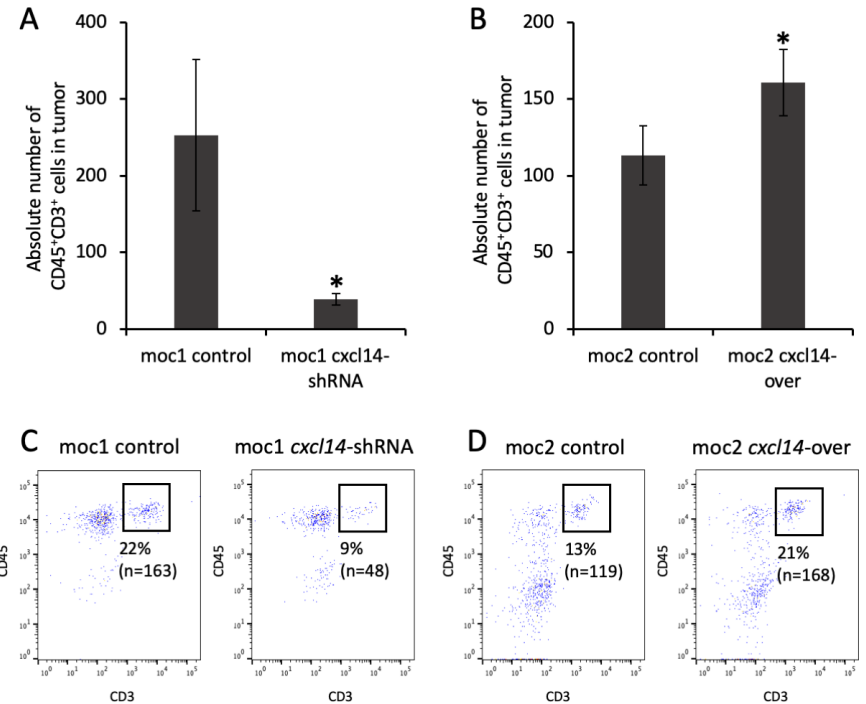

Figure 4 Modulation of CXCL14 is associated with direct changes in tumor infiltrating lymphocytes (TIL). (A) Bar plot shows significant decrease in absolute number of CD $45^{+} \mathrm{CD}^{+}$cells (cells $/ 1 \times 10^{5}$ ) in mouse oral cavity (MOC)1derived tumor after CXCL14 shRNA knockdown $(n=3$ biological replicates, 2 independent experiments, $p=0.02$, error bars represent SEM). (B) Bar plot shows significant increase in absolute number of CD $45^{+} \mathrm{CD} 3^{+}$cells (cells $/ 1 \times 10^{5}$ ) in MOC2-derived tumor after CXCL14 overexpression $(n=3$ biological replicates, 2 independent experiments, $p=0.006$, error bars represent SEM). (C) Fluorescent activated cell sorting (FACS) plots show decrease in proportion of intratumoral $\mathrm{CD} 45^{+} \mathrm{CD}^{+} \mathrm{T}$ cells from $22 \%$ in control MOC1derived tumor (left panel) to $9 \%$ after CXCL14 shRNA knockdown (right panel). (D) FACS plots show increase in proportion of intratumoral $\mathrm{CD} 45^{+} \mathrm{CD} 3^{+} \mathrm{T}$ cells from $13 \%$ in control MOC2-derived tumor (left panel) to $21 \%$ after CXCL14 overexpression (right panel).

reduction of $\mathrm{CD} 45^{+} \mathrm{CD} 3^{+} \mathrm{T}$ cells within the tumor relative to control (figure 4A,C, $\mathrm{p}=0.02, \mathrm{n}=3$ biological replicates). Conversely, tumors generated following CXCL14 overexpression in MOC2 cells demonstrated a $42 \%$ increase in $\mathrm{CD}_{4} 5^{+} \mathrm{CD}^{+} \mathrm{T}$ cells relative to control (figure 4B,D, $p=0.006, n=5$ biological replicates). Taken together, these results strongly suggest that the expression of CXCL14 in OSCC cells is associated with infiltration of T cells in vivo.

\section{CXCL14-related tumor growth changes are T cell dependent}

To assess whether the observed changes in tumor growth with CXCL14 modulation are dependent on the presence of TIL, we depleted T cells using a CD3 antibody and examined tumor growth. Strikingly, this resulted in a significantly diminished effect of CXCL14 modulation on MOC1-derived and MOC2-derived tumor growth (figure 5A,B), further supporting the conclusion that $\mathrm{T}$ cell infiltration underlies the tumor suppressive effect of CXCL14.

CXCL14 expression is associated with MHC class I expression It was previously shown that CXCL14 expression could restore MHC class I expression that was reduced by HPV in tumor cells. ${ }^{16}$ To determine the effect of CXCL14 on 

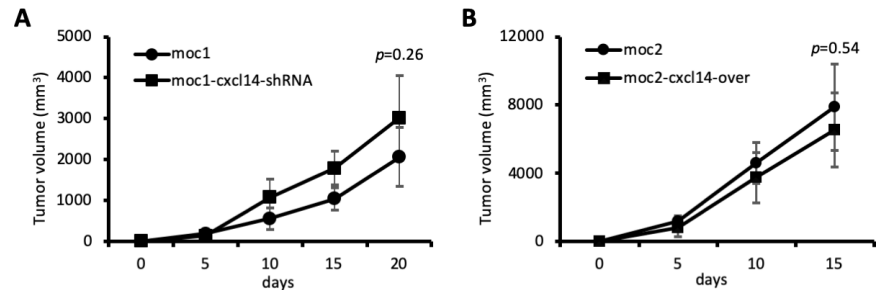

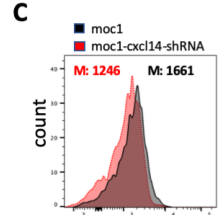

$\mathrm{H}-2 \mathrm{D}^{\mathrm{b}}$
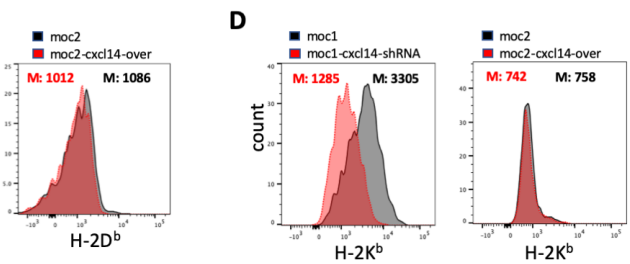

Figure 5 CXCL14-related changes in tumor growth are T cell dependent. (A) Line plot shows growth of mouse oral cavity (MOC)1-derived tumors with and without CXCL14 knockdown after depletion of T cells. (B) Line plot shows growth of MOC2-derived tumors with and without CXCL14 overexpression after depletion of T cells. The effect of CXCL14 modulation is significantly diminished and no longer reaches statistical significance. (C) Plots show fluorescence corresponding to cell surface expression of H-2Db. CXCL14 knockdown in MOC1 cells resulted in a significant decrease in $\mathrm{H}-2 \mathrm{Db}$ (left panel), while CXCL14 overexpression in MOC2 cells did not result in a significant change (right panel).

(D) Plots show fluorescence corresponding to cell surface expression of $\mathrm{H}-2 \mathrm{~Kb}$. CXCL14 knockdown in MOC1 cells resulted in a significant decrease in $\mathrm{H}-2 \mathrm{~Kb}$ (left panel), while CXCL14 overexpression in MOC2 cells did not result in a significant change (right panel).

MHC class I expression in our HPV-negative model, we assessed the impact of CXCL14 modulation on expression of $\mathrm{H}-2 \mathrm{D}^{\mathrm{b}}$ and $\mathrm{H}-2 \mathrm{~K}^{\mathrm{b}}$ on MOC1 and MOC2 cells. CXCL14 knockdown in MOC1 cells, which had high baseline expression of CXCL14, reduced the expression of both MHC class I molecules. However, in MOC2 cells, which had very low baseline expression of CXCL14, overexpression of CXCL14 did not affect MHC class I expression (figure 5C,D). To determine whether this association between CXCL14 and MHC class I holds in human samples, we evaluated the correlation between malignant cell CXCL14 and MHC class I genes in our scRNA-seq cohort. We identified a strong correlation in one patient sample (MEEI17), but not in other profiled samples (online supplemental figure S2).

\section{TIL are associated with malignant cell-specific CXCL14 expression in human samples}

To determine whether the association between CXCL14 expression and lymphocytic infiltrate is recapitulated in human patients, we first assessed stromal lymphocytes by histological analysis in our cohort of nine patients with OSCC analyzed by scRNA-seq and found a strong positive relationship between malignant cell CXCL14 expression and stromal lymphocytes (figure 6). We then assessed CXCL14 expression and TIL in two separate cohorts: (1) the same scRNA-seq cohort of 9 patients and (2) TCGA cohort of 498 patients with HNSCC. In our
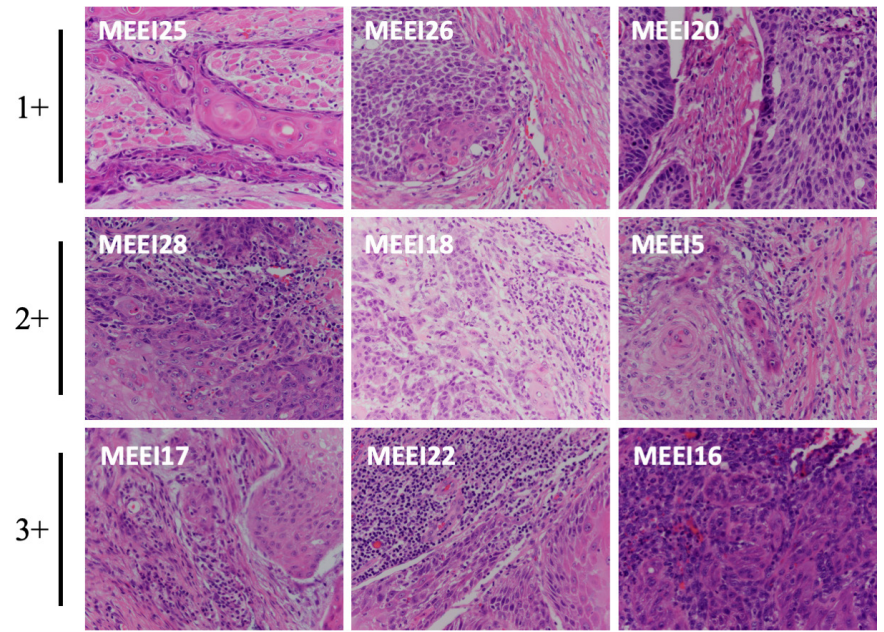

Figure 6 Malignant cell CXCL14 expression is associated with stromal lymphocytes. Representative H\&E images from nine primary tumors profiled by single cell RNA-sequencing show stromal lymphocytes quantified as 1+ in MEEl25, MEEI26 and MEEI20; 2+ in MEEI28, MEEl18 and MEEI5 and $3+$ in MEEI17, MEEI22 and MEEl16. Samples are ordered by CXCL14 expression in malignant cells.

scRNA-seq cohort, we found a strong significant association of TIL per HPF on H\&E with CXCL14 expression in malignant cells (figure 7A, $r=0.93, p=0.0003$ ), but not with CXCL14 expression in cancer-associated fibroblasts (figure 7B, $\mathrm{r}=-0.46, \mathrm{p}=0.26$ ) or CXCL14 expression in all non-malignant cells (figure $7 \mathrm{C}, \mathrm{r}=-0.13, \mathrm{p}=0.74$ ). Importantly, in the TCGA cohort, there was also no association of bulk CXCL14 expression with TIL as assessed by CD2 and CD3 expression (figure 7D, $\mathrm{r}=-0.04, \mathrm{p}=0.53$ ). Taken together, these results suggested a malignant cell-specific association of CXCL14 with TIL, with CXCL14 from other cellular sources, including fibroblasts, showing no association.

\section{DISCUSSION}

In this study, we demonstrate an association of TIL with CXCL14 expression in in vivo immunocompetent models of HPV-negative oral cavity cancer. In HPV-related HNSCC models, CXCL14 expression was previously associated with increased $\mathrm{T}$ cells in primary tumors ${ }^{16}$ and tumor draining lymph nodes. ${ }^{15}$ It was also associated with increased T cell activation and tumor cell MHC class I expression, resulting in tumor cell death, providing a mechanism for CXCL14-based tumor suppression. ${ }^{16}$ These studies lay essential ground work for our own studies, which extend these findings to the distinct pathologic entity of HPV-negative oral cavity cancer. We find concordant changes in tumor growth from CXCL14 shRNA knockdown in indolent MOC1 cells with high baseline expression of CXCL14 and CXCL14 overexpression in aggressive MOC2 cells with low baseline expression of CXCL14 (figure 3), leading to corresponding changes in TIL in both conditions (figure 4). Importantly, we demonstrate that the tumor suppressive effect 

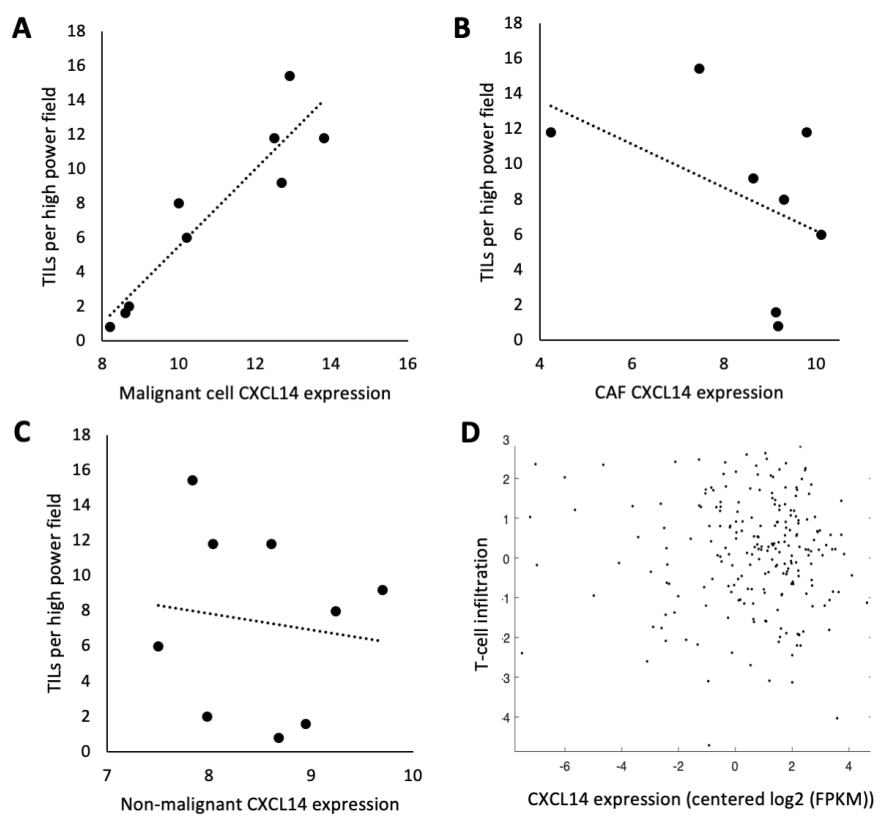

Figure 7 Malignant cell-specific CXCL14 expression is associated with tumor infiltrating lymphocytes (TIL). (A) Scatter plot shows highly significant direct correlation $(r=0.93, p=0.003$ ) between TILs per high power field (HPF) on H\&E and CXCL14 expression in malignant cells of nine tumors profiled by single cell RNA-sequencing (scRNA-seq).

(B) Scatter plot shows non-significant negative correlation $(r=-0.46, p=0.26$ ) between TILs per HPF on H\&E and CXCL14 expression in cancer-associated fibroblasts (CAFs) of nine tumors profiled by scRNA-seq. (C) Scatter plot shows no correlation $(r=-0.13, p=0.74)$ between TILs per HPF on H\&E and CXCL14 expression in all non-malignant cells of nine tumors profiled by scRNA-seq. For (A-C), CXCL14 expression was measured as $\log _{2}($ average(TPM)+1). (D) Scatter plot shows no significant correlation $(r=-0.04, p=0.53)$ between TILs measured by CD2 and CD3 expression levels and CXCL14 in bulk RNA-seq data in 498 TCGA head and neck squamous cell carcinoma (HNSCC) tumors.

of CXCL14 is dependent on the presence of TIL, with T cell depletion resulting in the loss of this effect (figure 5).

An important aspect of our work is the use of human tumor samples to further define the role of CXCL14 in the HNSCC ecosystem. By taking advantage of single cell RNA-seq to overcome the challenges of cellular and expression-based heterogeneity, ${ }^{19-21}$ our data suggest that the effects of CXCL14 in OSCC may specifically depend on the cell type secreting this chemokine, with only malignant cell-specific CXCL14 being associated with increased TIL in patient samples in our cohort (figure 7). Notably, this compartment-specific effect has been previously suggested in breast cancer: Sjöberg $e t a \hat{l}$ used RNAscope to demonstrate that epithelial CXCL14 was associated with decreased proliferation, while stromal CXCL14 was associated with shorter recurrence free and cancer-specific survival. This context dependence has also been supported by mouse models of breast cancer, in which fibroblast-derived CXCL14 led to increased tumor growth via NOS-dependent angiogenesis and macrophage recruitment, ${ }^{322}{ }^{23}$ while the opposite effect was seen with malignant cell-specific CXCL14. ${ }^{3}$ Such an effect has also been demonstrated with other cytokines in breast cancer. ${ }^{3}$ Interestingly, we do not find a significant correlation between malignant cell CXCL14 expression and expression of MHC class I genes, as demonstrated in mouse models. This lack of association may be related to more complex mechanisms of CXCL14-related tumor suppression or MHC class I regulation in human tumors, but they may also reflect the well known technical limitations of scRNA-seq, including alterations in gene expression due to tumor dissociation and cell sorting, as well as bias in genes detected. ${ }^{19}$

Our murine models of OSCC demonstrate the tumor suppressive effect of malignant cell-specific CXCL14; we anticipate that further work with manipulation of stromal and, specifically, fibroblast-derived CXCL14 would be useful in better defining this context dependence in HNSCG to determine if head and neck cancers mimic the findings from breast oncology. We believe our data validating inverse association of tumor size and direct association of TIL with malignant cell-specific CXCL14 expression may have implications for targeted therapies in OSCC and head and neck cancer, in general. Along with our data on TIL, the known epigenetic silencing of CXCL14 through promoter hypermethylation ${ }^{11}$ and recovery of its expression levels and function with 5-azacytidine ${ }^{12}$ suggest that reversal of this epigenetic modification may augment the efficacy of immunotherapy in head and neck cancer. Furthermore, the recovery of CXCL14 expression levels has been shown to improve cetuximab-dependent tumor suppression, suggesting an inverse relationship between CXCL14 and EGFR signaling. ${ }^{711}$ Additional investigation into safe and effective ways of recovering CXCL14 signaling within head and neck cancer may mature CXCL14 as a viable therapeutic target, especially for prevention of delayed regional failure in cervical nodal basins of the neck.

\section{CONCLUSIONS}

In analysis of human single cell RNA-seq data, we find that CXCL14 is the most significantly differentially downregulated gene in malignant cells within metastatic lymph nodes, relative to corresponding primary HNSCC tumors. In murine immunocompetent models of HPVnegative OSCC, we demonstrate that CXCL14 expression in vivo is associated with TIL, with overexpression associated with increased TIL and decreased tumor growth and knockdown associated with decreased TIL and increased tumor growth. We find that growth changes in response to CXCL14 modulation are T cell dependent. In human patient samples, we further demonstrate that only malignant cell-specific CXCL14 is associated with TIL, a context dependence that has previously been demonstrated in breast cancer but not in OSCC. These data support the tumor suppressive role of CXCL14 in OSCC via induction of TIL and provide the first direct in vivo patient data to support the malignant cell specificity of this effect. 


\section{Author affiliations}

${ }^{1}$ Otolaryngology, Massachusetts Eye and Ear Infirmary, Boston, Massachusetts, USA

${ }^{2}$ Otolaryngology, Stanford University School of Medicine, Stanford, California, USA

${ }^{3}$ Pathology, Massachusetts Eye and Ear Infirmary, Boston, Massachusetts, USA

${ }^{4}$ Department of Molecular Cell Biology, Weizmann Institute of Science, Rehovot, Israel

${ }^{5}$ Otolaryngology, Washington University in Saint Louis School of Medicine, Saint Louis, Missouri, USA

${ }^{6}$ Genetics, Washington University School of Medicine, St. Louis, M0, USA

Contributors ASP, JHS, JBS, and SVP were responsible for study concepts, study design, data analysis and interpretation and manuscript preparation. ASP, JHS, WCF, DTL and IT were responsible for data acquisition and statistical analysis. ASP, JHS, WCF, DTL, IT, JBS and SVP were responsible for manuscript editing and review.

Funding Supported by V Foundation (SVP), Cancer Research Foundation (SVP), Barnes Jewish Hospital Foundation (SVP) and NCl 1K08CA237732 (SVP).

Disclaimer The funding sources had no involvement in the design, conduct, and reporting of the research.

Competing interests None declared.

Patient consent for publication Not required

Ethics approval All animal procedures were performed in accordance with protocols approved by the Administrative Panel on Laboratory Animal Care at Stanford University (Stanford, California, USA). Histopathological analysis of patient samples was reviewed by the Massachusetts Eye and Ear institutional review board and deemed to be of minimal risk.

Provenance and peer review Not commissioned; externally peer reviewed.

Data availability statement Data are available in a public, open access repository. The human single cell RNA-sequencing data analyzed are available through dbGaP (accession number phs001474.v1.p1)

Supplemental material This content has been supplied by the author(s). It has not been vetted by BMJ Publishing Group Limited (BMJ) and may not have been peer-reviewed. Any opinions or recommendations discussed are solely those of the author(s) and are not endorsed by BMJ. BMJ disclaims all liability and responsibility arising from any reliance placed on the content. Where the content includes any translated material, BMJ does not warrant the accuracy and reliability of the translations (including but not limited to local regulations, clinical guidelines, terminology, drug names and drug dosages), and is not responsible for any error and/or omissions arising from translation and adaptation or otherwise.

Open access This is an open access article distributed in accordance with the Creative Commons Attribution Non Commercial (CC BY-NC 4.0) license, which permits others to distribute, remix, adapt, build upon this work non-commercially, and license their derivative works on different terms, provided the original work is properly cited, appropriate credit is given, any changes made indicated, and the use is non-commercial. See http://creativecommons.org/licenses/by-nc/4.0/.

\section{ORCID iD}

Anuraag Parikh http://orcid.org/0000-0003-0968-1028

\section{REFERENCES}

1 Hara T, Tanegashima K. Pleiotropic functions of the CXC-type chemokine CXCL14 in mammals. J Biochem 2012;151:469-76.

2 Wang W, Huang P, Zhang L, et al. Antitumor efficacy of C-X-C motif chemokine ligand 14 in hepatocellular carcinoma in vitro and in vivo. Cancer Sci 2013:104:1523-31.
3 Sjöberg E, Augsten M, Bergh J, et al. Expression of the chemokine CXCL14 in the tumour stroma is an independent marker of survival in breast cancer. Br J Cancer 2016;114:1117-24.

4 Lin K, Zou R, Lin F, et al. Expression and effect of CXCL14 in colorectal carcinoma. Mol Med Rep 2014;10:1561-8.

5 Song EY, Shurin MR, Tourkova IL, et al. Epigenetic mechanisms of promigratory chemokine CXCL14 regulation in human prostate cancer cells. Cancer Res 2010;70:4394-401.

6 Wente MN, Mayer C, Gaida MM, et al. CXCL14 expression and potential function in pancreatic cancer. Cancer Lett 2008;259:209-17.

7 Nakayama R, Arikawa K, Bhawal UK. The epigenetic regulation of CXCL14 plays a role in the pathobiology of oral cancers. $J$ Cancer 2017;8:3014-27.

8 Ozawa S, Kato Y, Komori R, et al. BRAK/CXCL14 expression suppresses tumor growth in vivo in human oral carcinoma cells. Biochem Biophys Res Commun 2006;348:406-12.

9 Izukuri K, Suzuki K, Yajima N, et al. Chemokine CXCL14/BRAK transgenic mice suppress growth of carcinoma cell transplants. [corrected]. Transgenic Res 2010;19:1109-17.

10 Hata R-I, Izukuri K, Kato Y, et al. Suppressed rate of carcinogenesis and decreases in tumour volume and lung metastasis in CXCL14/ BRAK transgenic mice. Sci Rep 2015;5:9083.

11 Kondo T, Ozawa S, Ikoma T, et al. Expression of the chemokine CXCL14 and cetuximab-dependent tumour suppression in head and neck squamous cell carcinoma. Oncogenesis 2016;5:e240.

12 Tessema M, Klinge DM, Yingling CM, et al. Re-expression of CXCL14, a common target for epigenetic silencing in lung cancer, induces tumor necrosis. Oncogene 2010;29:5159-70.

13 Shellenberger TD, Wang M, Gujrati M, et al. BRAK/CXCL14 is a potent inhibitor of angiogenesis and a chemotactic factor for immature dendritic cells. Cancer Res 2004;64:8262-70.

14 Shurin GV, Ferris R, Tourkova IL, et al. Loss of new chemokine CXCL14 in tumor tissue is associated with low infiltration by dendritic cells (DC), while restoration of human CXCL14 expression in tumor cells causes attraction of DC both in vitro and in vivo. $J$ Immunol 2006;176:3840.2

15 Cicchini L, Westrich JA, Xu T, et al. Suppression of antitumor immune responses by human papillomavirus through epigenetic downregulation of CXCL14. mBio 2016;7:1-13.

16 Westrich JA, Vermeer DW, Silva A, et al. CXCL14 suppresses human papillomavirus-associated head and neck cancer through antigenspecific $\mathrm{CD} 8^{+} \mathrm{T}$-cell responses by upregulating $\mathrm{MHC}$-I expression. Oncogene 2019;38:7166-80.

17 Puram SV, Tirosh I, Parikh AS, et al. Single-Cell transcriptomic analysis of primary and metastatic tumor ecosystems in head and neck cancer. Cell 2017;171:1611-24.

18 Frederick MJ, Henderson Y, Xu X, et al. In vivo expression of the novel CXC chemokine BRAK in normal and cancerous human tissue. Am J Pathol 2000;156:1937-50.

19 Qi Z, Barrett T, Parikh AS, et al. Single-Cell sequencing and its applications in head and neck cancer. Oral Oncol 2019;99:104441.

20 Stepan KO, Li MM, Kang SY, et al. Molecular margins in head and neck cancer: current techniques and future directions. Oral Oncol 2020;110:104893.

21 Puram SV, Parikh AS, Tirosh I. Single cell RNA-seq highlights a role for a partial EMT in head and neck cancer. Mol Cell Oncol 2018:5:e1448244.

22 Augsten M, Hägglöf C, Olsson E, et al. CXCL14 is an autocrine growth factor for fibroblasts and acts as a multi-modal stimulator of prostate tumor growth. Proc Natl Acad Sci U S A 2009;106:3414-9

23 Augsten M, Sjöberg E, Frings O, et al. Cancer-associated fibroblasts expressing CXCL14 rely upon NOS1-derived nitric oxide signaling for their tumor-supporting properties. Cancer Res 2014:74:2999-3010. 